\title{
Palladium-Catalyzed Annulation of Arynes by 2-Halobenzaldehydes: Synthesis of
}

\section{Fluoren-9-ones}

Xiaoxia Zhang and Richard C. Larock ${ }^{*}$

Department of Chemistry, Iowa State University, Ames, Iowa 50011

\section{Supporting Information}

General. The ${ }^{1} \mathrm{H}$ and ${ }^{13} \mathrm{C}$ NMR spectra were recorded at 300 and $75.5 \mathrm{MHz}$ or 400 and $100 \mathrm{MHz}$ respectively. All melting points are uncorrected. High resolution mass spectra were recorded on a Kratos MS50TC double focusing magnetic sector mass spectrometer using EI at $70 \mathrm{eV}$. All reagents were used directly as obtained commercially unless otherwise noted.

Synthesis of Starting Materials. Compounds 1, 2, 4 and 11 were purchased from Sigma-Aldrich Co. Compounds $5,{ }^{1} \mathbf{1 4},{ }^{2} \mathbf{1 7}^{3}$ and $\mathbf{1 9}^{4}$ were prepared according to literature procedures.

Compounds 7, 9 and 12 were prepared from the respective commercially available 2-bromobenzaldehydes by the previously reported three-step procedure shown below. ${ }^{5}$ Compound 7 was obtained as a light yellow solid in a $75 \%$ overall yield.
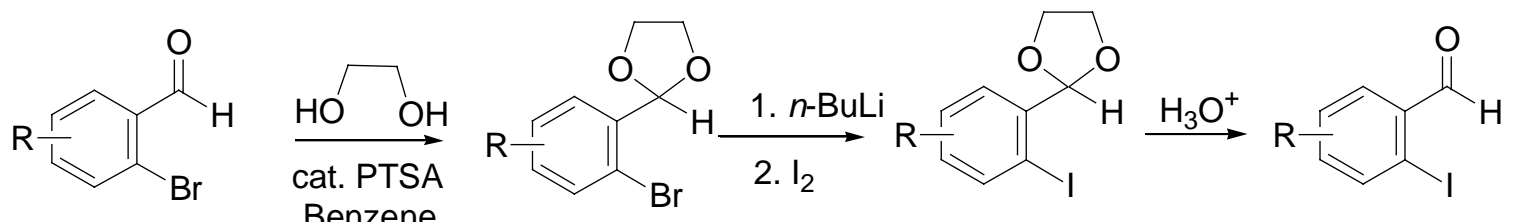

The spectral properties were identical with those previously reported. ${ }^{5}$ Compound $\mathbf{9}$ was obtained as a white solid in an $80 \%$ overall yield. The spectral properties were identical with those previously reported. ${ }^{6}$ 
1-Iodo-2-naphthalenecarboxaldehyde (12). Compound 12 was obtained as a light yellow solid in a $60 \%$ overall yield: $\mathrm{mp} 106-107{ }^{\circ} \mathrm{C} ;{ }^{1} \mathrm{H} \mathrm{NMR}\left(\mathrm{CDCl}_{3}, 300 \mathrm{MHz}\right) \delta$ 7.57-7.60 (m, 2H), 7.70-7.73 (m, 2H), 7.78-7.81 (d, $J=8.7 \mathrm{~Hz}, 1 \mathrm{H}), 8.31-8.35(\mathrm{~m}, 1 \mathrm{H})$; ${ }^{13} \mathrm{C} \mathrm{NMR}\left(\mathrm{CDCl}_{3}, 300 \mathrm{MHz}\right) \delta 124.96,128.71,128.83,129.50,129.94,133.45,134.03$, 134.61, 137.10, 197.72; IR $\left(\mathrm{CHCl}_{3}\right) 1680 \mathrm{~cm}^{-1}$; HRMS m/z 281.9545 (calcd for $\mathrm{C}_{11} \mathrm{H}_{7} \mathrm{IO}$, 281.9542).

General Procedure for the Palladium-Catalyzed Synthesis of Fluoren-9-ones. The 2-iodobenzaldehyde $(0.30 \mathrm{mmol})$, the 2-(trimethylsilyl)aryl triflate $(1.50 \mathrm{mmol}), \mathrm{CsF}$ (1.50 mmol), $\mathrm{Pd}(\mathrm{dba})_{2}(0.015 \mathrm{mmol}), \mathrm{P}(o \text {-tolyl })_{3}(0.015 \mathrm{mmol}), 2 \mathrm{~mL}$ of toluene, and 2 $\mathrm{mL}$ of $\mathrm{MeCN}$ were placed in a 4 dram vial and the vial was sealed. The reaction mixture was stirred first at room temperature for $1 \mathrm{~min}$ and then heated to $110{ }^{\circ} \mathrm{C}$ for $12 \mathrm{~h}$. The mixture was allowed to cool to room temperature (CAUTION: OPENING THE VIAL AT HIGH TEMPERATURE CAN BE DANGEROUS!), diluted with diethyl ether, washed with brine, dried over anhydrous $\mathrm{MgSO}_{4}$, and concentrated under reduced pressure. The product was isolated by flash chromatography on silica gel.

Compounds $\mathbf{3},{ }^{7} \mathbf{1 3},{ }^{8} \mathbf{1 8},{ }^{9} \mathbf{2 0},{ }^{9}$ and $\mathbf{2 1}{ }^{9}$ were obtained after flash chromatography, and their spectral properties are identical with these previously reported.

1-Fluoro-9H-fluoren-9-one (6). The indicated compound was obtained as a yellow solid in an $82 \%$ yield. The reaction mixture was chromatographed using 10:1 hexane/EtOAc: $\operatorname{mp} 117-119{ }^{\circ} \mathrm{C} ;{ }^{1} \mathrm{H}$ NMR $\left(\mathrm{CDCl}_{3}, 300 \mathrm{MHz}\right) \delta 6.89-6.95(\mathrm{t}, J=8.4 \mathrm{~Hz}$, $1 \mathrm{H}), 7.29-7.35(\mathrm{~m}, 2 \mathrm{H}), 7.43-7.53(\mathrm{~m}, 3 \mathrm{H}), 7.65-7.67(\mathrm{~d}, J=7.5 \mathrm{~Hz}, 1 \mathrm{H}) ;{ }^{13} \mathrm{C} \mathrm{NMR}$ $\left(\mathrm{CDCl}_{3}, 75 \mathrm{MHz}\right) \delta 116.61,116.65,117.59,117.87,120.12,120.29,120.88,124.69$ $129.90,134.19,134.86,137.23,137.34,143.59,143.63,146.56,146.61,157.79,161.29$, 
190.36 (extra peaks due to F splitting); IR $\left(\mathrm{CHCl}_{3}\right)$ 2923, 1716, 1600, $1471 \mathrm{~cm}^{-1}$; HRMS $m / z$ 198.0485 (calcd for $\mathrm{C}_{13} \mathrm{H}_{7} \mathrm{FO}, 198.0481$ ).

9H-Fluoreno[2,3- $\boldsymbol{d}][\mathbf{1 , 3}]$ dioxol-9-one $(\mathbf{8}) . \quad$ The reaction mixture was chromatographed using 10:1 hexane/EtOAc and the indicated compound was obtained as a yellow solid in a $56 \%$ yield: $\mathrm{mp} 145-147{ }^{\circ} \mathrm{C} ;{ }^{1} \mathrm{H}$ NMR $\left(\mathrm{CDCl}_{3}, 400 \mathrm{MHz}\right) \delta 6.05(\mathrm{~s}$, 2H), $6.95(\mathrm{~s}, 1 \mathrm{H}), 7.09(\mathrm{~s}, 1 \mathrm{H}), 7.18-7.22(\mathrm{~m}, 1 \mathrm{H}), 7.31-7.33(\mathrm{~d}, J=7.6 \mathrm{~Hz}, 1 \mathrm{H}), 7.39-$ $7.43(\mathrm{~m}, 1 \mathrm{H}), 7.53-7.55(\mathrm{~d}, J=7.6 \mathrm{~Hz}, 1 \mathrm{H}) ;{ }^{13} \mathrm{C} \mathrm{NMR}\left(\mathrm{CDCl}_{3}, 100 \mathrm{MHz}\right) \delta 101.76$ 102.27, 105.18, 119.31, 123.81, 128.39, 128.71, 134.38, 134.76, 141.74, 143.67, 148.50, 153.37, 192.45; IR $\left(\mathrm{CHCl}_{3}\right)$ 1709, 1593, 1477, $1458 \mathrm{~cm}^{-1} ; \mathrm{HRMS} \mathrm{m} / 2224.0476$ (calcd for $\left.\mathrm{C}_{14} \mathrm{H}_{8} \mathrm{O}_{3}, 224.0473\right)$.

$10 H$-Fluoreno[1,2- $\boldsymbol{d}][\mathbf{1 , 3}]$ dioxol-10-one $(\mathbf{1 0}) . \quad$ The reaction mixture was chromatographed using 20:1 hexane/EtOAc and the indicated compound was obtained as a yellow solid in a 72\% yield (from the aryl iodide) or $50 \%$ (from the aryl bromide): $\mathrm{mp}$ 161-163 ${ }^{\circ} \mathrm{C} ;{ }^{1} \mathrm{H}$ NMR $\left(\mathrm{CDCl}_{3}, 400 \mathrm{MHz}\right) \delta 6.12(\mathrm{~s}, 2 \mathrm{H}), 6.81(\mathrm{~d}, J=7.6 \mathrm{~Hz}, 1 \mathrm{H}), 6.97$ (d, $J=7.6 \mathrm{~Hz}, 1 \mathrm{H}), 7.24-7.26(\mathrm{~m}, 1 \mathrm{H}), 7.45-7.46(\mathrm{~m}, 2 \mathrm{H}), 7.65(\mathrm{~d}, J=7.6 \mathrm{~Hz}, 1 \mathrm{H}) ;{ }^{13} \mathrm{C}$ NMR $\left(\mathrm{CDCl}_{3}, 100 \mathrm{MHz}\right) \delta 103.05,111.96,113.60,115.44,119.94,124.41,128.21$, 134.24, 134.62, 136.89, 144.60, 145.00, 150.44, 190.92; IR $\left(\mathrm{CHCl}_{3}\right)$ 1710, 1468, 1250 $\mathrm{cm}^{-1}$; HRMS m/z 224.0478 (calcd for $\mathrm{C}_{14} \mathrm{H}_{8} \mathrm{O}_{3}, 224.0473$ ).

2,3-Dimethyl-9H-fluoren-9-one (15). The reaction mixture was chromatographed using 20:1 hexane/EtOAc and the indicated compound was obtained as a yellow solid in a $71 \%$ yield: $\mathrm{mp} 88-89{ }^{\circ} \mathrm{C} ;{ }^{1} \mathrm{H} \mathrm{NMR}\left(\mathrm{CDCl}_{3}, 400 \mathrm{MHz}\right) \delta$ 7.22-7.25 (m, 2H), 7.41-7.43 (m, 3H), $7.59(\mathrm{~d}, J=7.6 \mathrm{~Hz}, 1 \mathrm{H}) ;{ }^{13} \mathrm{C} \mathrm{NMR}\left(\mathrm{CDCl}_{3}, 100 \mathrm{MHz}\right) \delta 19.95$, 20.77, 119.84, 121.82, 124.11, 125.59, 128.55, 132.34, 134.41, 134.64, 137.62, 142.55, 
144.19, 144.65, 194.12 IR $\left(\mathrm{CHCl}_{3}\right)$ 2922, 1707, 1601, $1452 \mathrm{~cm}^{-1} ; \mathrm{HRMS} \mathrm{m/z} 208.0891$ (calcd for $\mathrm{C}_{15} \mathrm{H}_{12} \mathrm{O}, 208.0888$ ).

1-Fluoro-6,7-dimethyl-9H-fluoren-9-one (16). The reaction mixture was chromatographed using 20:1 hexane/EtOAc and the indicated compound was obtained as a yellow solid in a $73 \%$ yield: mp $165-167{ }^{\circ} \mathrm{C} ;{ }^{1} \mathrm{H} \mathrm{NMR}\left(\mathrm{CDCl}_{3}, 400 \mathrm{MHz}\right) \delta 2.24(\mathrm{~s}$, $3 \mathrm{H}), 2.29(\mathrm{~s}, 3 \mathrm{H}), 6.82-6.87(\mathrm{t}, J=8.8 \mathrm{~Hz}, 1 \mathrm{H}), 7.17(\mathrm{~d}, J=7.6 \mathrm{~Hz}, 1 \mathrm{H}), 7.21(\mathrm{~s}, 1 \mathrm{H})$, 7.36-7.39 (m, 2H); ${ }^{13} \mathrm{C} \mathrm{NMR}\left(\mathrm{CDCl}_{3}, 100 \mathrm{MHz}\right) \delta 19.99,20.73,115.92,115.95,116.88$, $117.09,120.20,120.32,122.07,125.60,132.10,136.70,136.78,138.39,141.48,141.51$, 144.24, 146.62, 146.66, 157.91, 160.52, 190.35 (extra peaks are due to F-splitting); IR $\left(\mathrm{CHCl}_{3}\right)$ 2920, 1713, 1618, $1454 \mathrm{~cm}^{-1} ; \mathrm{HRMS} \mathrm{m/z} 226.0798$ (calcd for $\mathrm{C}_{15} \mathrm{H}_{11} \mathrm{FO}$, 226.0794).

\section{References}

(1) Bridges, A. J.; Lee, A.; Maduakor, E. C.; Schwartz, C. E. Tetrahedron Lett. 1992, 33,7499 .

(2) Yoshida, H.; Sugiura, S.; Kunai, A. Org. Lett. 2002, 4, 2767.

(3) Peña, D.; Pérez, D.; Guitián, E.; Castedo, L. J. Am. Chem. Soc. 1999, 121, 5827.

(4) Yoshikawa, E.; Radhakrishnan, K. V.; Yamamoto, Y. J. Am. Chem. Soc. 2000, 123,7280 .

(5) Bogucki, D. E.; Charlton, J. L. J. Org. Chem. 1995, 60, 588.

(6) Mattson, R. J.; Sloan, C. P.; Lockhart, C. C.; Catt, J. D.; Gao, Q.; Huang, S. J. Org. Chem. 1999, 64, 8004.

(7) This compound was identified by comparing the ${ }^{1} \mathrm{H}$ and ${ }^{13} \mathrm{C}$ NMR spectra and melting point with an authentic sample obtained from Aldrich Chemical Co., Inc. 
(8) Campo M. A.; Larock, R. C. Org. Lett. 2000, $2,3675$.

(9) Campo M. A.; Larock, R. C. J. Org. Chem. 2002, 67, 5616. 


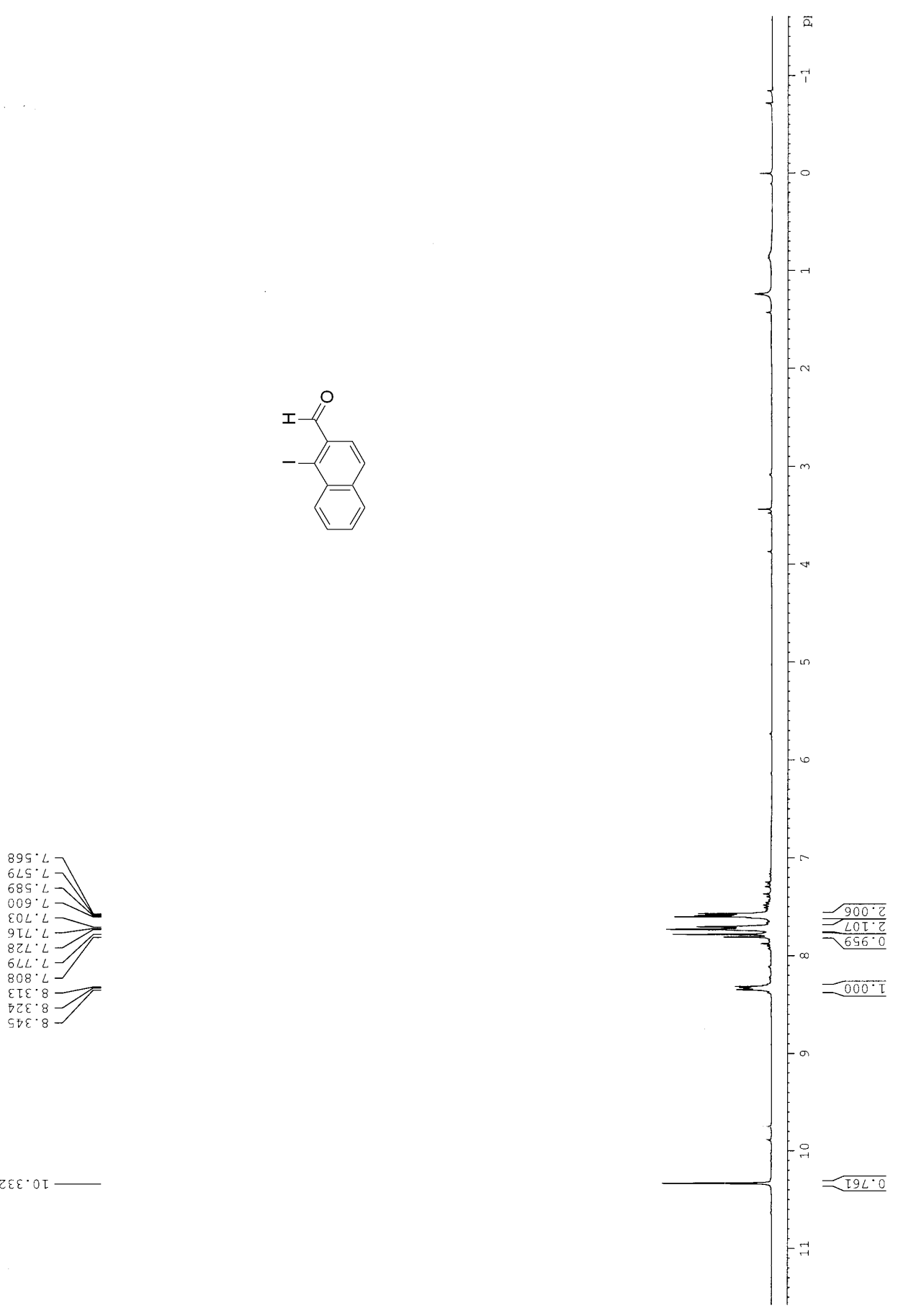



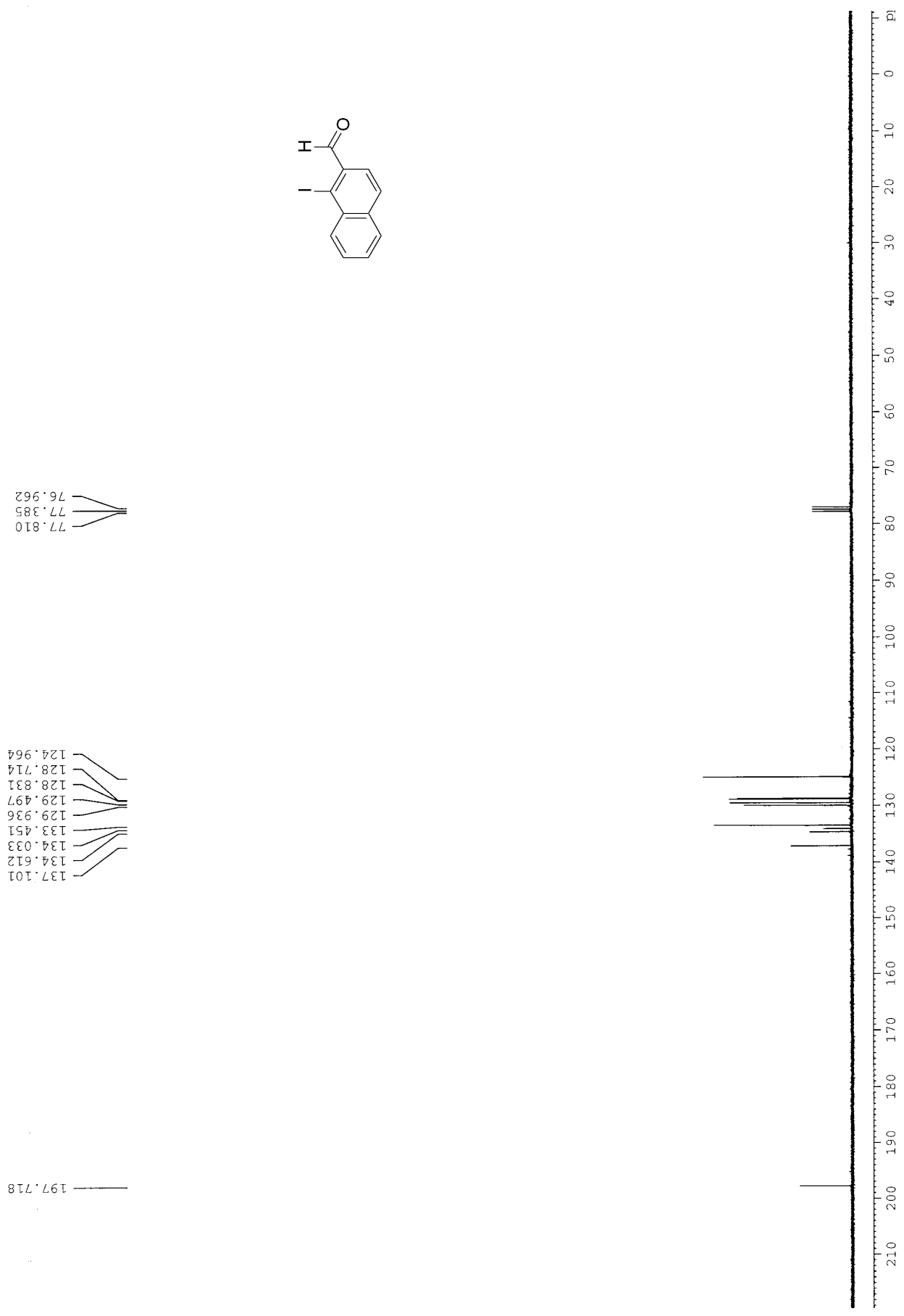

is

$8 I L \cdot L 6 I-$ 


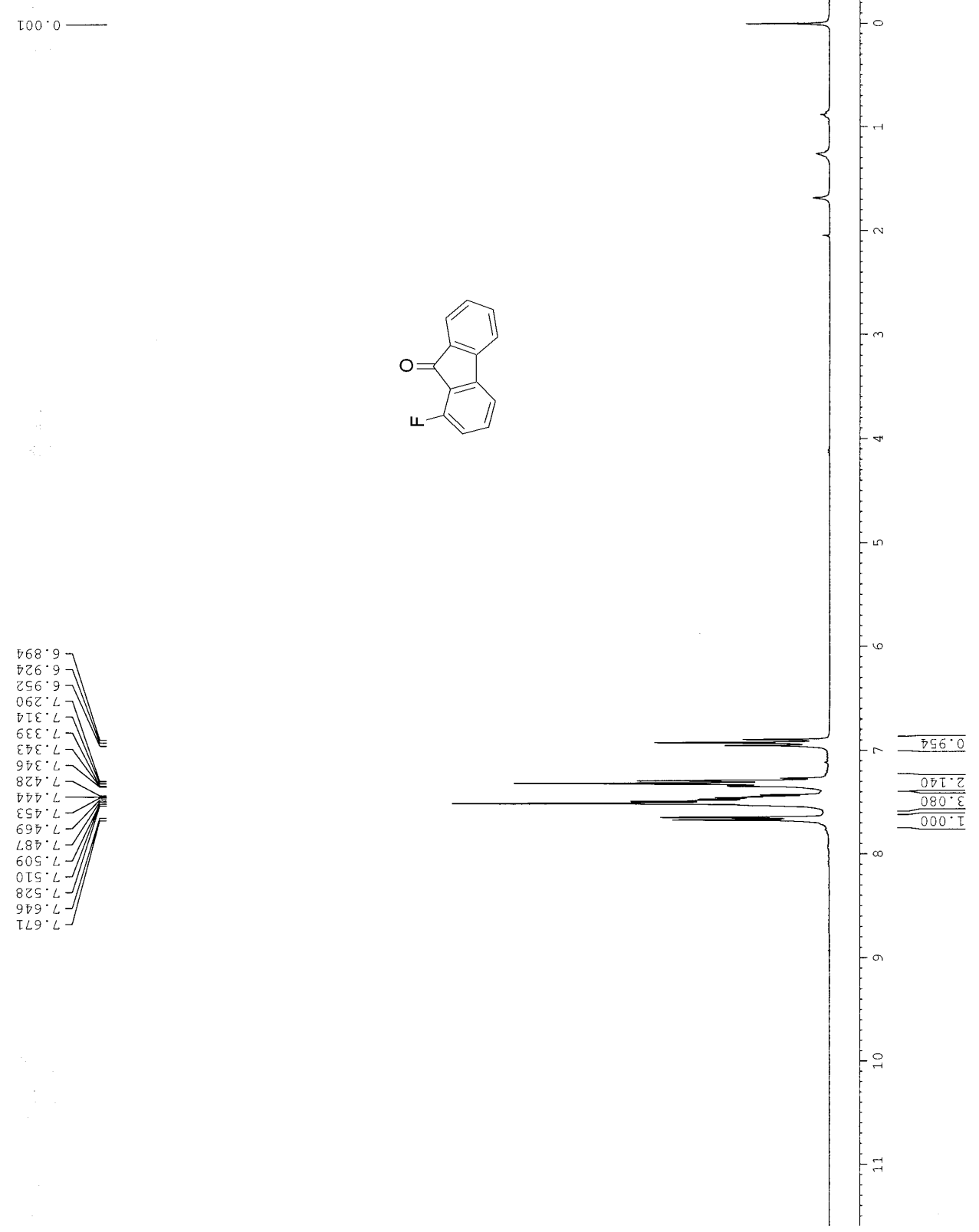




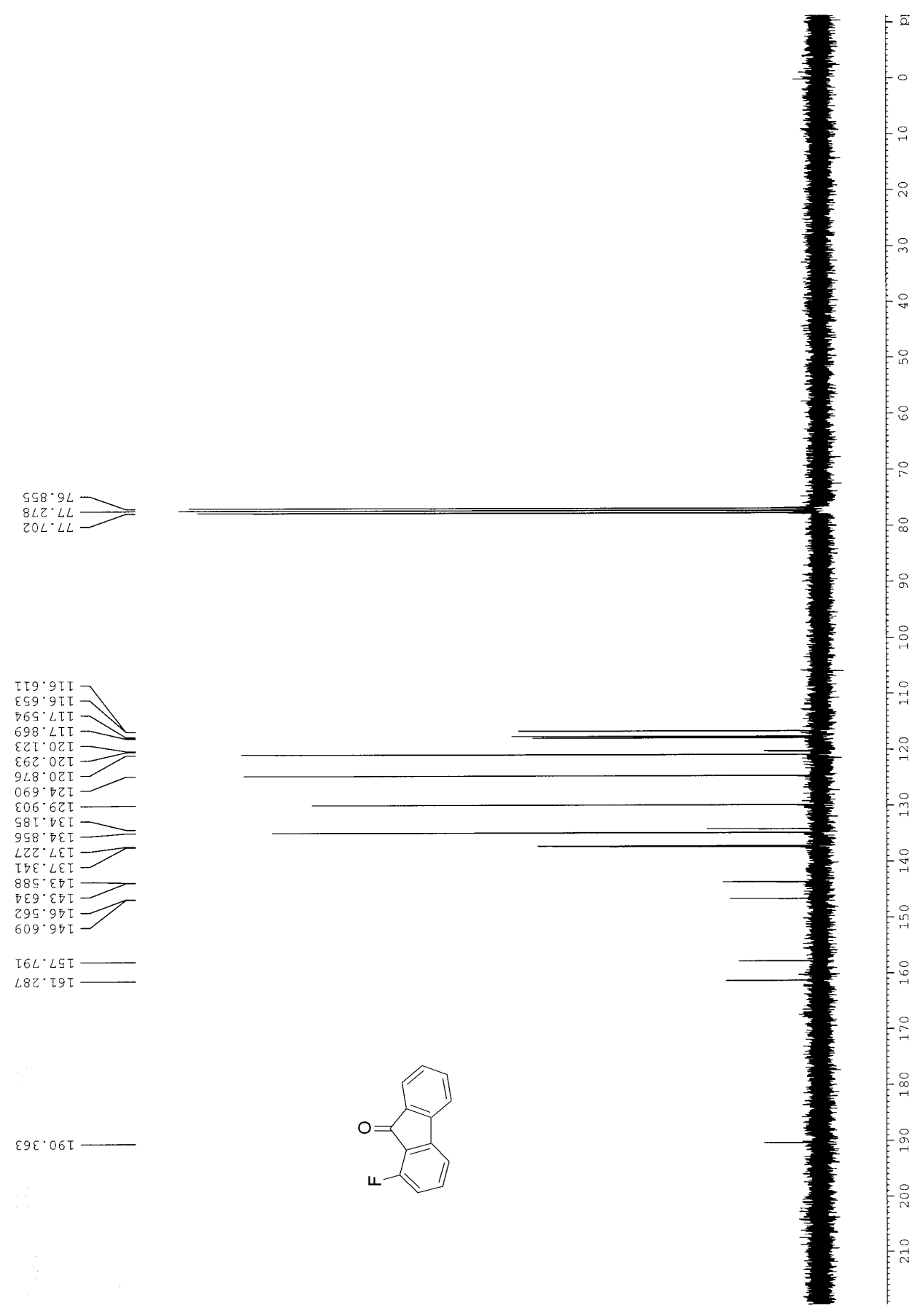

จ 


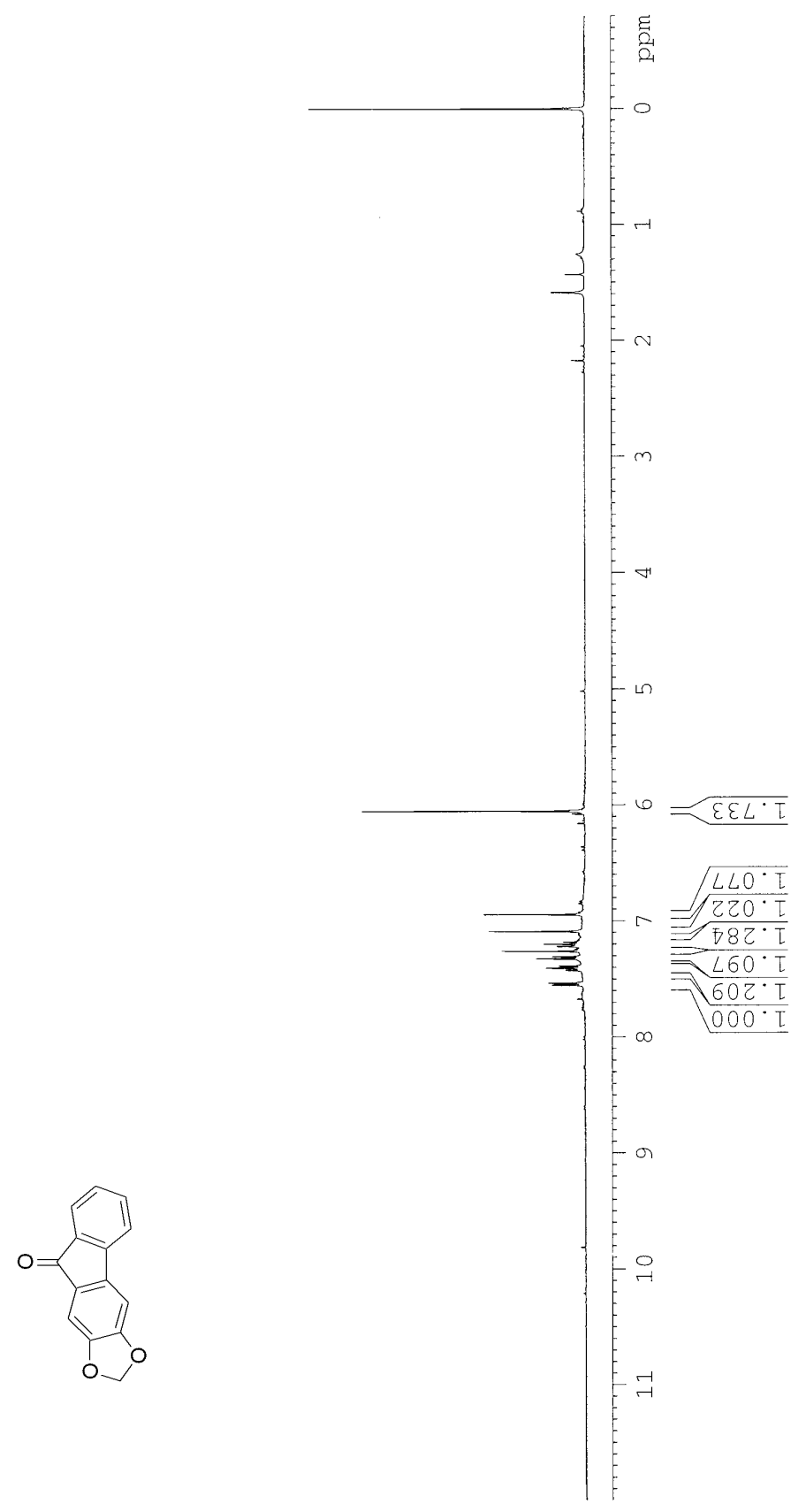

$\frac{0}{\sim}$

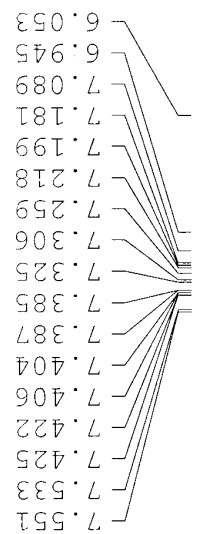




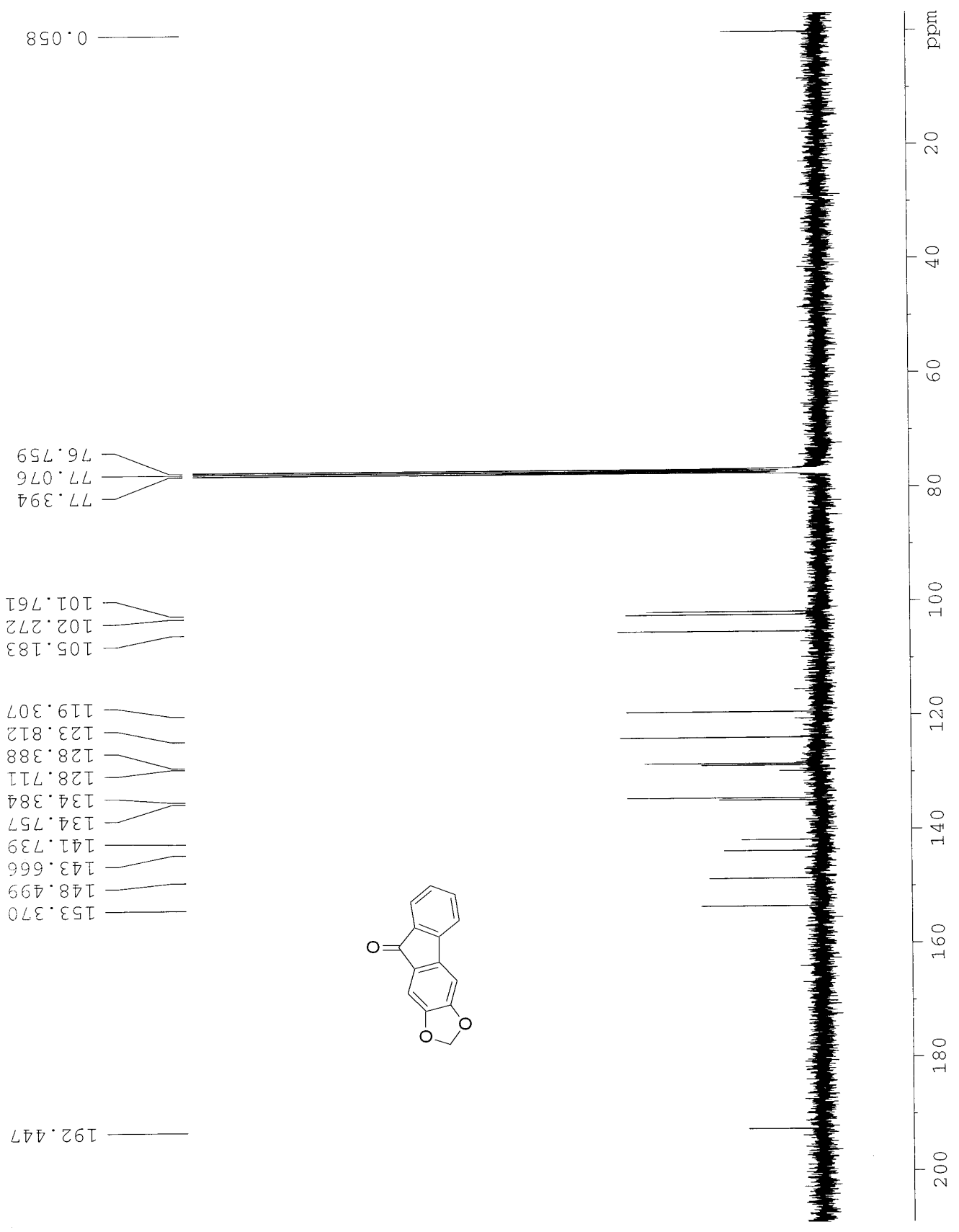




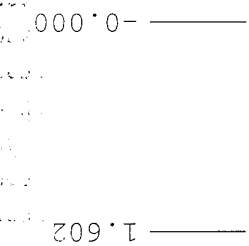

$2.09^{\circ} \mathrm{I}$

$\ldots$
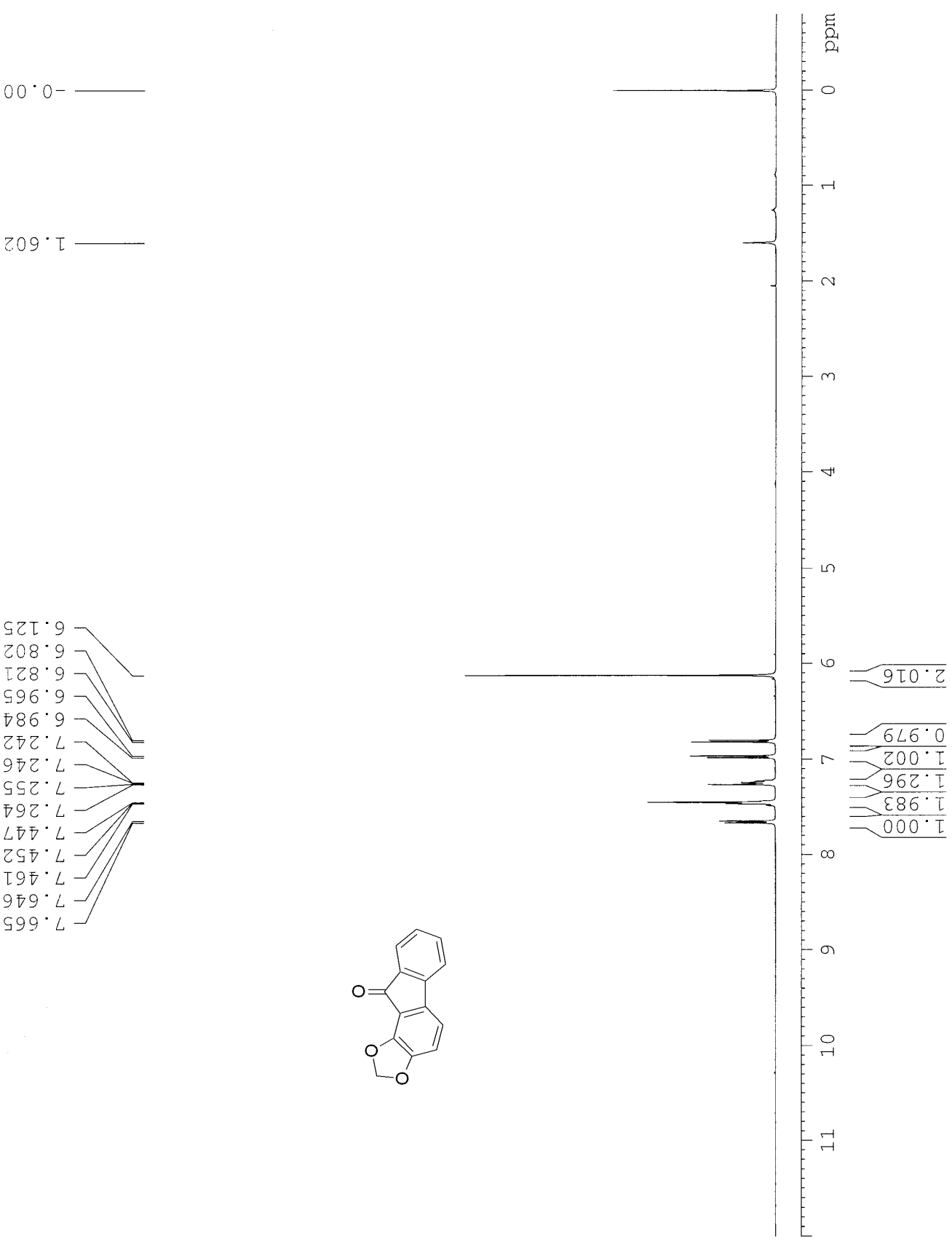


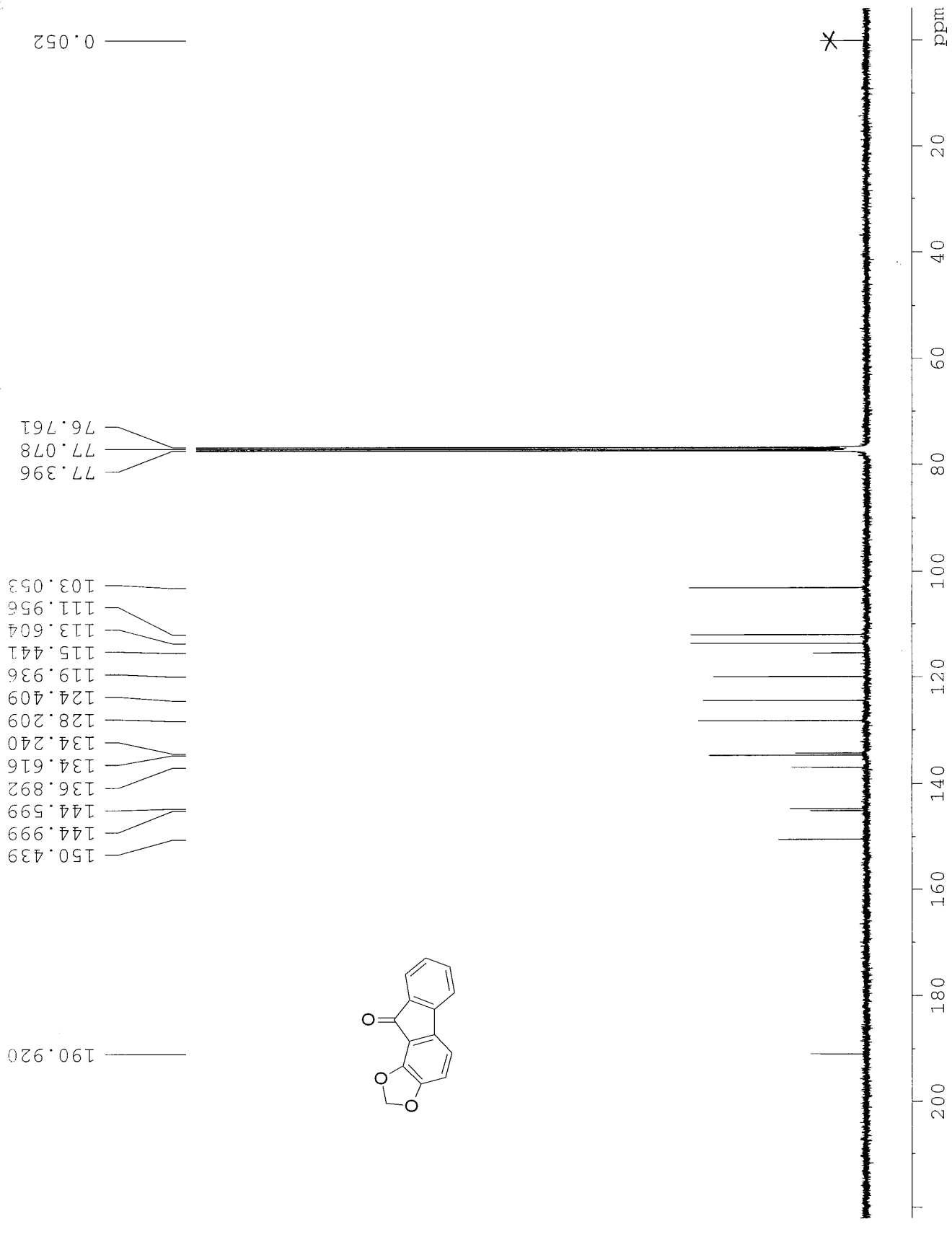



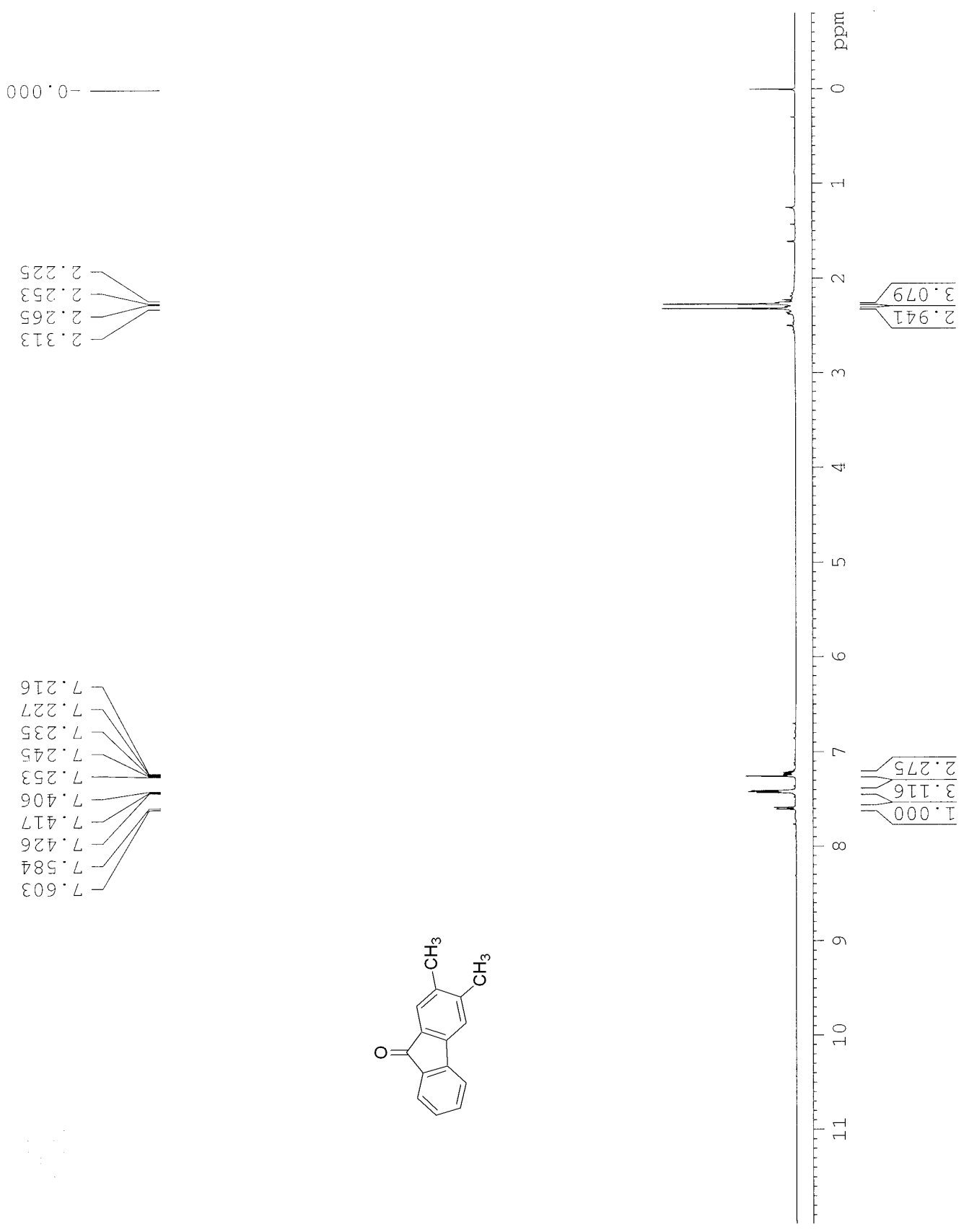


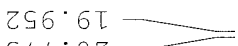

$\varepsilon \angle L \cdot O z$

$6 \subseteq L \cdot 9 L$
$9 L 0^{\circ} \angle L$
$76 \varepsilon \cdot L L$

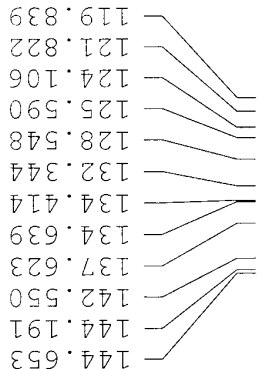

2己I'

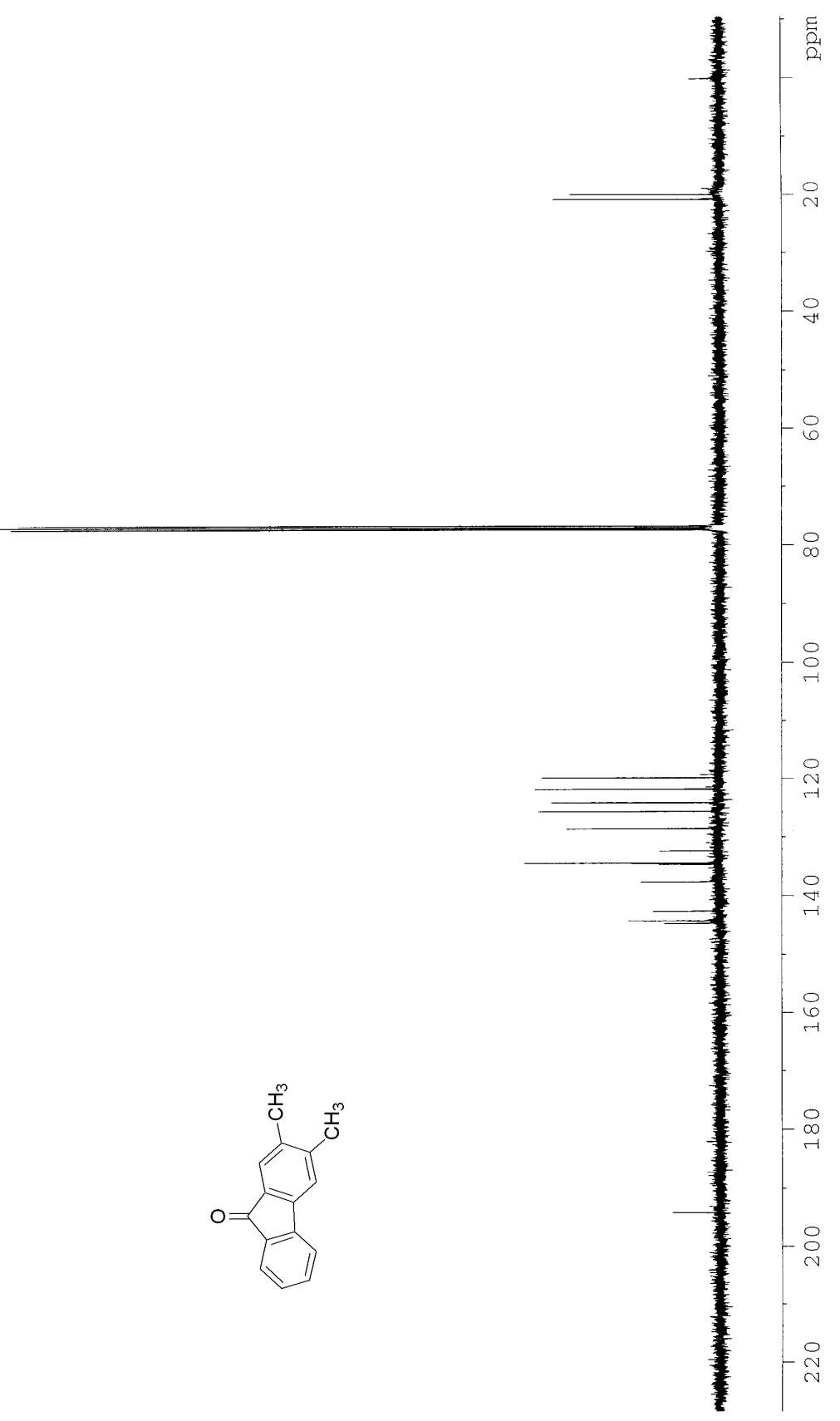



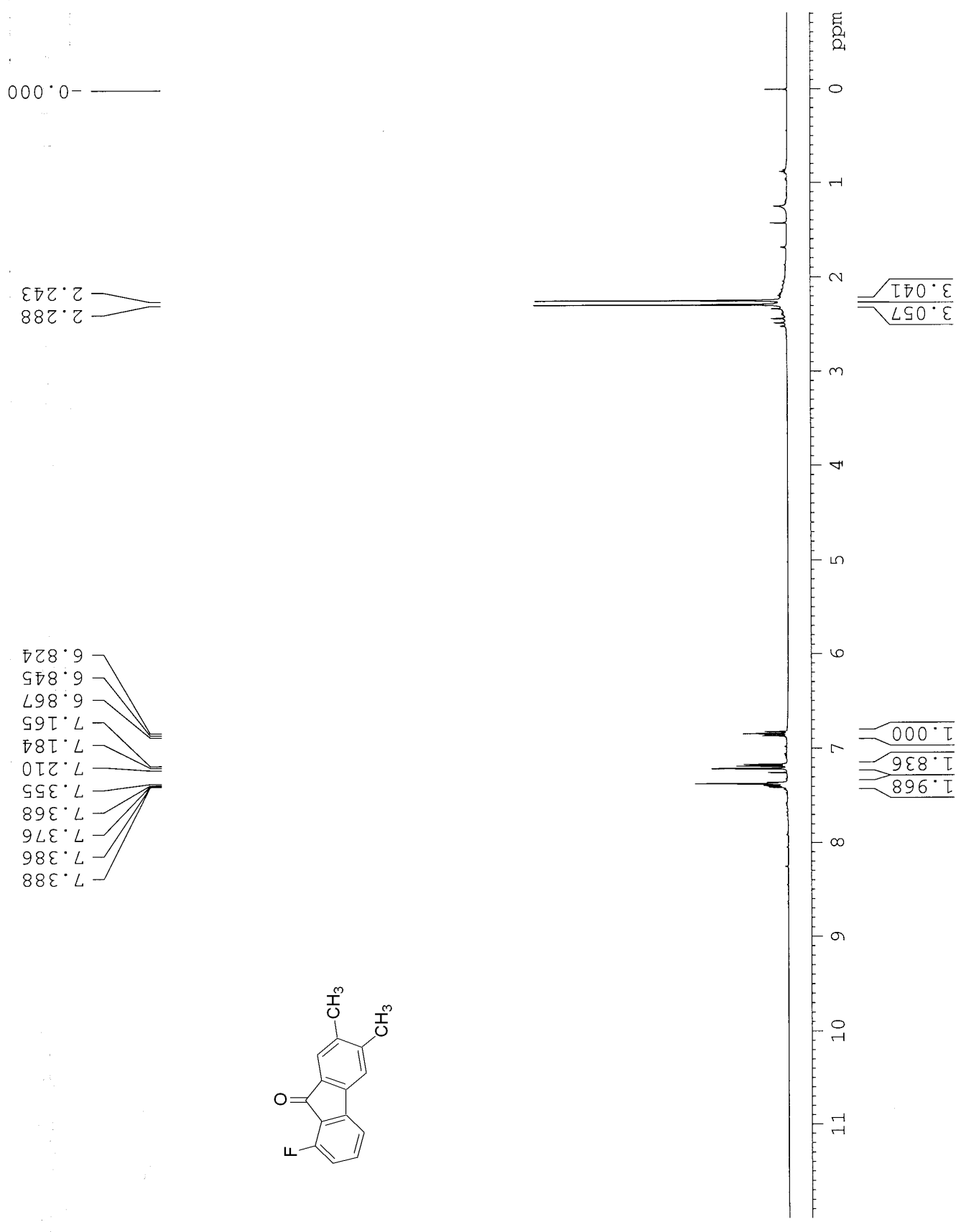

ธ28.9

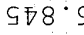

$\angle 98 \cdot 9$

S9I. $L>$

ธ8T'L

$0 โ T^{\circ} L$

ऽร $\varepsilon^{\circ} L$

$89 \varepsilon^{\circ} L$

$9\left\llcorner\varepsilon^{\circ} L\right.$

$98 \varepsilon^{\circ} L$

$88 \varepsilon^{\circ} L$ 

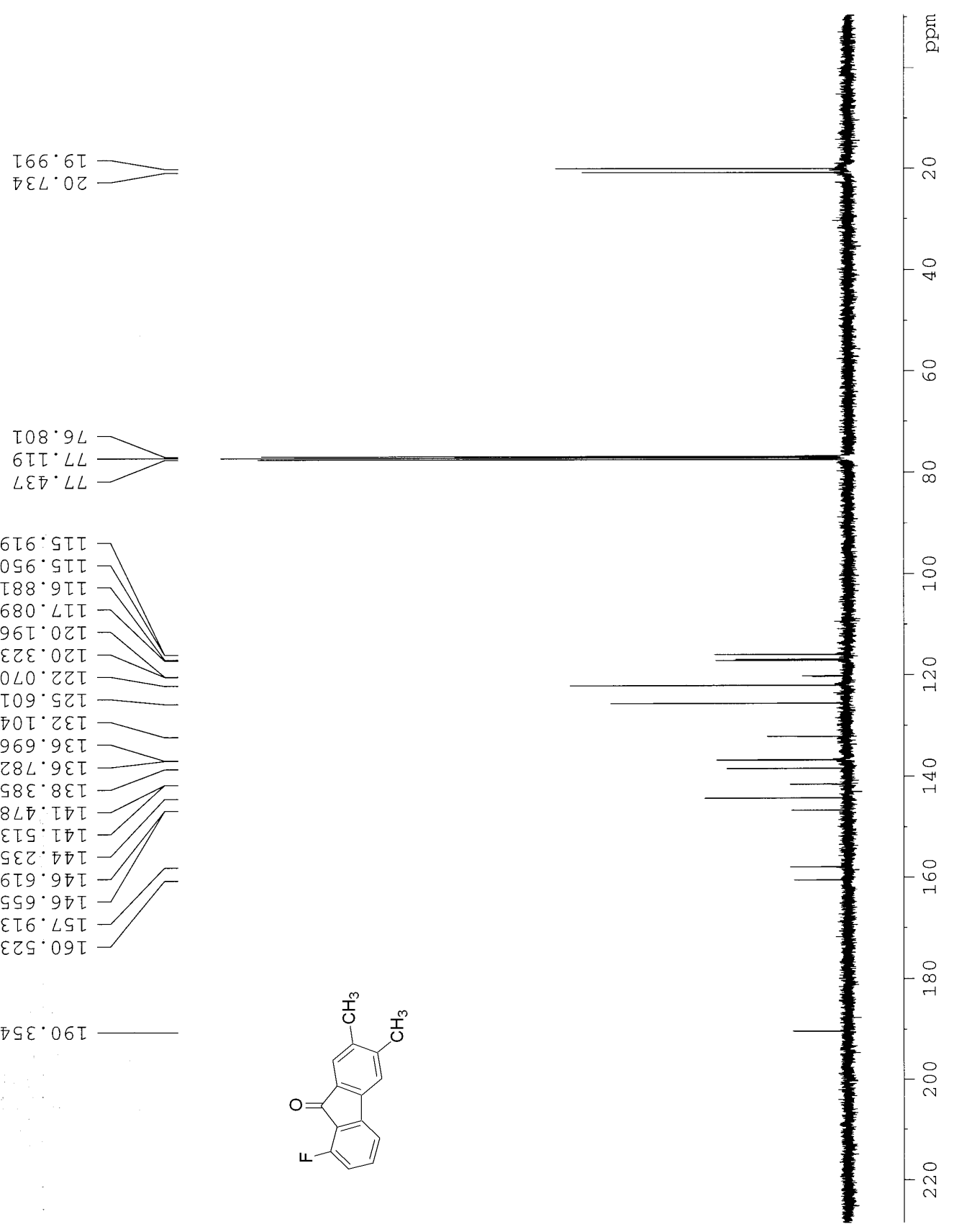

$\frac{1}{n}$

$6 T 6: S I T$
$0 S 6$

โ88.9

$680^{\circ}$ LIT

$96 \mathrm{I}^{\circ} \mathrm{\circ} \mathrm{ZT}$

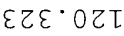

$0 \angle 0^{\circ} \mathrm{ZZT} \longrightarrow$

TO9. $92 \mathrm{DL}$

ธ०โ' $2 \varepsilon T$

$969 \cdot 9 \varepsilon \tau=$

$28 L \cdot 9 \varepsilon \tau \longrightarrow$

ธ8ร.8हโ

$8 \angle \nabla \cdot \tau \nabla \tau-$

EโG $\cdot \tau \tau \tau-$

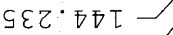

6โ9.9ரI

$559 \cdot 97 \mathrm{~T}$

ET6. LST

$\varepsilon 7 \mathrm{~S}^{\circ} \cdot 09 \mathrm{~L}$

†૬ร.06โ 